\title{
Atmospheric occultation of optical intersatellite links: coherence loss and related parameters
}

\author{
Nicolas Perlot \\ German Aerospace Center (DLR), Institute of Communications and Navigation, Wessling, \\ Germany (nicolas.perlot@dlr.de)
}

Received 20 October 2008; revised 19 March 2009; accepted 29 March 2009; posted 30 March 2009 (Doc. ID 102961); published 14 April 2009

\begin{abstract}
The Rytov theory is applied to find the wave structure function of a laser beam transmitted from one satellite to another and propagating through the turbulent atmosphere. The phase-screen approximation is used. Taking into account refractive-index anisotropy, outer scale, and atmospheric mean-refraction defocusing, we provide expressions of the wave structure function for a spherical wave. The width and time of coherence at the receiver are evaluated. Expression for the beam spread is found using the extended Huygens-Fresnel principle, and beam wander is assessed. Beam wander occurs only for very narrow beams. Links involving low-Earth-orbit and geosynchronous satellites are studied as examples. Finally, conditions where optical tracking is perturbed by the atmosphere are examined. (C) 2009 Optical Society of America

OCIS codes: $\quad 010.1330,030.0030,060.2605$.
\end{abstract}

\section{Introduction}

The laser technology applied to intersatellite links (ISLs) has gained reliability over the past several decades. Optical ISLs are meant for either communications or measurements. When near-Earth satellites are involved, link occultation by the Earth and by its atmosphere can be frequent. About thirty years ago, several theoretical studies derived spatial and temporal spectra of phase and intensity perturbations during planetary occultation [1-3]. Considered scenarios were essentially spacecraft radio occultation or stellar occultation. In those studies, anisotropic inhomogeneities and mean refraction of the quiescent atmosphere were already taken into account. Several occultation experiments have been conducted (with the Earth or other planets) and, with various degrees of agreement, theory could be compared to measurements [4-8]. However, so far the main investigated turbulence effect has been scintillation.

Development of optical space communications allows now the measurement of occulted narrow

0003-6935/09/122290-13\$15.00/0

(C) 2009 Optical Society of America quasi-monochromatic beams. For such scenarios, other effects related to the loss of spatial coherence require investigation. For example, during an Earth occultation of an optical link between the OICETS and ARTEMIS satellites, the optical tracking system was challenged by high angle-of-arrival fluctuations [9]. In this paper, we evaluate the wave structure function (WSF) based on recent turbulence models. The goal is to estimate various parameters that are commonly evaluated in beam propagations at ground. These parameters are the coherence width and coherence time, the beam-spread loss, and the beam-wander strength. The paper is mostly derivative in the sense that it applies the well known Rytov theory and the extended Huygens-Fresnel principle. Unlike intensity statistics, phase statistics governed by the Rytov approximation have an acceptable validity in the strong-fluctuation regime. In the paper, emphasis is put on links involving low-Earthorbit (LEO) and geosynchronous (GEO) satellites. Although an optical beam crossing the atmosphere at altitudes below $10 \mathrm{~km}$ is likely to be blocked by clouds, the atmosphere is assumed free of clouds at any altitude.

The paper is structured as follows. Section 2 presents the model of optical turbulence from which 
wave perturbations are derived. Section 3 describes the link assumptions and the geometrical parameters used throughout the paper. Assuming a spherical wave, Sections 4 and 5 evaluate the wave structure function in the spatial and temporal domains. In Section 6 a finite beam radius is considered, and beam spread and beam wander are evaluated. Section 7 comments on the possible deterioration of optical tracking. Section $\underline{8}$ concludes on the results.

\section{Optical-Turbulence Model}

Following Gurvich et al. [10-12], we consider a threedimensional refractive-index spectrum $\Phi_{n}(\kappa)$ that is the sum of an isotropic part $\Phi_{n, i}(\kappa)$ and an anisotropic part $\Phi_{n, a}(\kappa)$ :

$$
\Phi_{n}(\kappa)=\Phi_{n, i}(\kappa)+\Phi_{n, a}(\kappa) .
$$

In both spectrum parts, inner scale is ignored whereas a finite outer scale is considered.

\section{A. Isotropic Spectrum}

We consider the von Karman spectrum given by

$$
\Phi_{n, i}(\kappa)=0.033 C_{n}^{2}\left(\kappa^{2}+\kappa_{0, i}^{2}\right)^{-11 / 6},
$$

with $\kappa_{0, i}=2 \pi / L_{0, i}$ and $L_{0, i}$ the isotropic outer scale. In our analysis, we define the $C_{n}^{2}$ parameter so that it fits the Hufnagel-Valley model in the lower atmosphere and the measurements of Gurvich [12] in the upper atmosphere. The total $C_{n}^{2}$ profile over the altitude $h$ takes the following form:

$$
C_{n}^{2}(h)=\mathrm{HV}_{5 / 7}(h)+\langle N(h)\rangle^{2} C_{K}(h) .
$$

$\mathrm{HV}_{5 / 7}$ is the widely used Hufnagel-Valley profile, which, for a zenith downlink at a wavelength $\lambda=$ $500 \mathrm{~nm}$, leads to a Fried parameter of $5 \mathrm{~cm}$ and an anisoplanatic angle of $7 \mu \mathrm{rad}$ (see Table 3.1 in Ref. [13]). $\langle N(h)\rangle$ is the mean refractivity. Assuming a wavelength $\lambda>300 \mathrm{~nm}$, the dependence of $N$ on $\lambda$ is negligible and $\langle N(h)\rangle$ is proportional to the air density. We consider $\langle N(h)\rangle=2.7 \times 10^{-4} e^{-h / H_{0}}$, where the characteristic height is set to $H_{0}=7 \mathrm{~km}$. The $C_{K}$ parameter is given by

$$
C_{K}(h)=10^{-10} e^{h / 10 \mathrm{~km}} \quad\left[\mathrm{~m}^{-2 / 3}\right] .
$$

Equation (4) fits the experimental values of Ref. [12] in the range $25 \mathrm{~km}<h<50 \mathrm{~km}$. The measurement campaign with the MIR space station is not the only one revealing that, for the stratosphere, the exponential fall off of $\mathrm{HV}_{5 / 7}$ is probably too steep $[9,12,14]$. Robert et al. [15] also relied on a hybrid $C_{n}^{2}$ model similar to Eq. (3) for comparison with measurements. The upper graph of Fig. 1 shows $C_{n}^{2}(h)$ together with the two constituting models for low and high atmospheres. Note that $\langle N(h)\rangle^{2} C_{K}(h)$ has little influence on the vertical profile integration. For a zenith down-
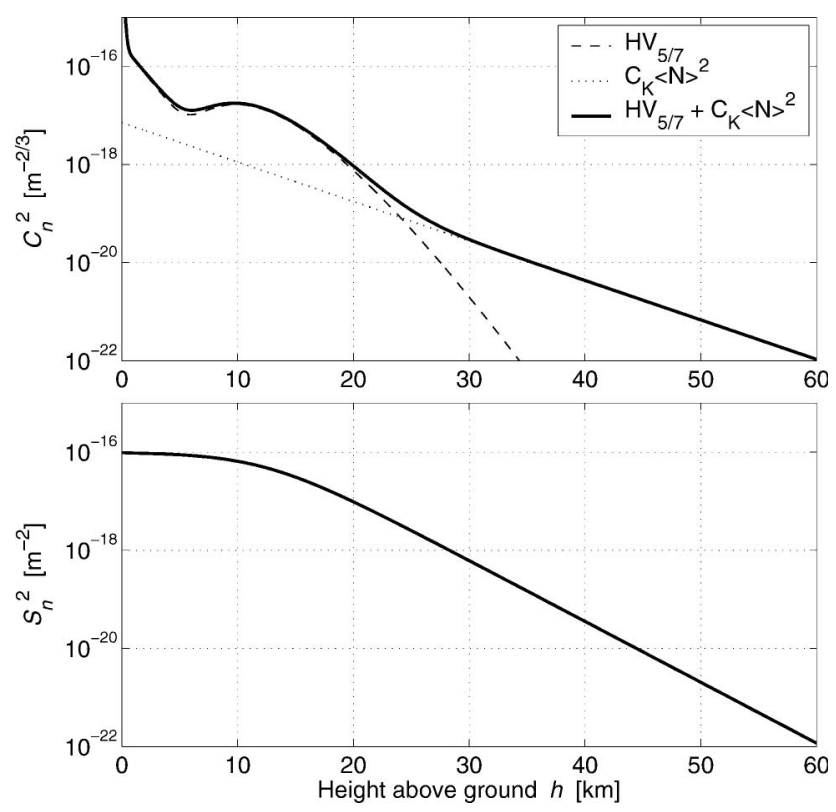

Fig. 1. Considered atmospheric profiles of turbulence: $C_{n}^{2}(h)$ and $S_{n}^{2}(h)$.

link at $\lambda=500 \mathrm{~nm}$, Eq. (3) leads to a Fried parameter of $4.9 \mathrm{~cm}$ and an anisoplanatic angle of $6.3 \mu \mathrm{rad}$.

\section{B. Anisotropic Spectrum}

We consider the following anisotropic refractiveindex spectrum $[10,12]$ :

$$
\Phi_{n, a}(K)=S_{n}^{2}\left(K^{2}+\kappa_{0, a}^{2}\right)^{-5 / 2},
$$

where $S_{n}^{2}$ is the scaling parameter of the spectrum, $\kappa_{0, a}=2 \pi / L_{0, a}$ with $L_{0, a}$ the anisotropic vertical outer scale, and

$$
K^{2}=\eta^{2}\left(K_{X}^{2}+K_{Y}^{2}\right)+K_{Z}^{2}
$$

$K_{X}$ and $K_{Y}$ are the horizontal (with respect to the Earth surface) spatial frequency variables, and $K_{Z}$ is the spatial frequency variable in the vertical direction (i.e., along the line passing through the Earth center). Following other authors, the anisotropy factor $\eta$ is set to $30[\underline{12}, \underline{15}]$. The parameter $S_{n}^{2}$ is given by

$$
S_{n}^{2}=\eta^{2}\langle N\rangle^{2} C_{W}
$$

Measurements of the parameter $C_{W}(h)$ in the range $25 \mathrm{~km}<h<50 \mathrm{~km}$ are reported in [12]. We use the following function:

$$
C_{W}(h)=5 \times 10^{-11} \frac{1}{1+4.5 \times 10^{8}\langle N\rangle^{2}} \quad\left[\mathrm{~m}^{-2}\right] .
$$

Thus $C_{W}(h)$ equals $5 \times 10^{-11} \mathrm{~m}^{-2}$ for high $h$ values and decreases when $h$ goes under $15 \mathrm{~km}$ so that $S_{n}^{2}$ saturates toward $10^{-16} \mathrm{~m}^{-2}$. The parameter $S_{n}^{2}(h)$ is plotted in the lower graph of Fig. 1. Although there is no undisputed model for the outer scale, it 
generally grows with altitude. We will assume the following exponential increase:

$$
L_{0, a}(h) \propto e^{h / 10 \mathrm{~km}} \quad[\mathrm{~m}] .
$$

Note that the function $0.1 \times e^{h / 10 \mathrm{~km}}$ is a good fit of the measured anisotropic inner scale [12], which is noted $l_{W}$ by Gurvich and Chunchuzov.

\section{Link Assumptions}

\section{A. Link Geometry}

Figure 2 shows the geometrical parameters of an intersatellite link crossing the Earth's atmosphere. $H_{P}$ is the altitude of the perigee corresponding to the shortest distance between the link path and the Earth's surface. $H_{1}$ (respectively, $H_{2}$ ) is the altitude of the transmitter (respectively, receiver). Along the beam path, we consider different link segments. $L_{1}$ (respectively, $L_{2}$ ) is the distance from the transmitter (respectively, receiver) to the perigee. $L$ is the link distance $\left(L=L_{1}+L_{2}\right)$. The Earth is assumed spherical with $R_{e}=6370 \mathrm{~km}$. Because $H_{P} \ll R_{e}, L$ is independent of $H_{P}$.

Because the refractive index has anisotropic properties, the axes $x$ and $y$ transverse to the beam direction must be clearly defined and not confused with the geocentric axes $(X, Y, Z)$. As shown in Fig. 3 , the $x, y, z$ axes form an orthogonal basis. The $x$ axis is parallel to the Earth vertical at the point $z=L_{1}$ and is directed outwardly from the Earth. A parameter that is also relevant is the grazing angle $\alpha$, which is the angular difference between the beam direction and the local Earth horizon.

We will often refer to two satellites of common orbits, namely, a circular low Earth orbit (LEO) and a geosynchronous orbit (GEO). The considered parameters for these two satellites are listed in Table $\underline{1}$.

\section{B. Phase-Screen Approximation}

We wish to estimate $L_{\text {atm }}$, which is half of the link's atmospheric range. In order to accurately model the atmosphere as a thin phase screen (PS), $L_{\text {atm }}$ should be significantly smaller than $L_{1}$ and $L_{2}$. We define the path-integrated $C_{n}^{2}$ by

$$
\mu_{0, i} \equiv \int_{0}^{L} C_{n}^{2}[h(z)] \mathrm{d} z .
$$

As depicted in Fig. $3, z$ is the longitudinal variable of the link with the transmitter as origin. An estimation of $L_{\text {atm }}$ can be found by writing

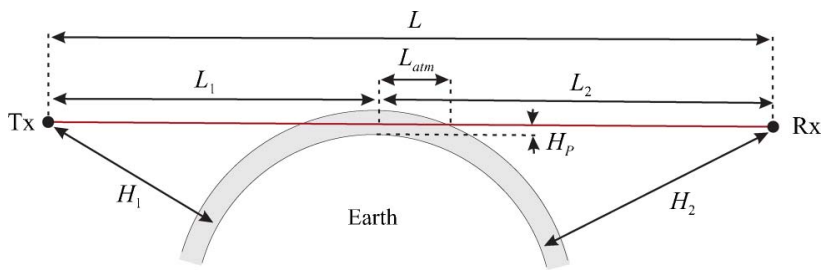

Fig. 2. (Color online) Considered link geometry.

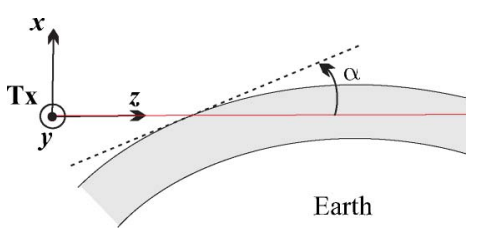

Fig. 3. (Color online) Beam coordinate system and $\alpha$ parameter.

$$
\int_{L_{1}-L_{\mathrm{atm}}}^{L_{1}+L_{\mathrm{atm}}} C_{n}^{2}[h(z)] \mathrm{d} z=p \mu_{0, i}
$$

where $p$ is the percentage of turbulence in the path segment $\left|z-L_{1}\right|<L_{\text {atm }}$ with respect to the total path $0<z<L$. With $h \ll R_{e}$, the variables $h$ and $z$ are related by $\left(z-L_{1}\right)^{2} \approx 2\left(h-H_{P}\right) R_{e}$. By additionally considering the typical trend $C_{n}^{2}(h) \sim \exp \left(-2 h / H_{0}\right)$, Eq. (11) leads us to

$$
L_{\mathrm{atm}} \approx \operatorname{erfinv}(p) \sqrt{H_{0} R_{e}},
$$

where erfinv is the inverse error function. Setting $p=0.99$, we find $L_{\text {atm }} \approx 380 \mathrm{~km}$. The atmospheric height span $H_{\text {span }}$ over which the ray propagates is given by $H_{\text {span }} \approx L_{\text {atm }}^{2} /\left(2 R_{e}\right)$, which yields $H_{\text {span }} \approx$ $11 \mathrm{~km}$. Note that $H_{\text {span }}$ is approximately the characteristic height of $L_{0, a}(h)$, so that the outer scale can be assumed constant over the whole path without introducing large errors. Finally, within the link segment of significant turbulence, one can evaluate the maximum grazing angle $\alpha_{\max }$ according to $\alpha_{\max } \approx$ $L_{\text {atm }} / R_{e}$, which yields $\alpha_{\max } \approx 3.4^{\circ}$.

\section{Beam Model}

The beam considered is a quasi-monochromatic single-mode collimated Gaussian beam. We assume the beam is in the far field as it enters the atmosphere, i.e.,

$$
L_{R} \ll L_{1},
$$

$L_{R}$ being the Rayleigh range. This condition is fulfilled for most realistic optical links. For example, with a beam of wavelength $\lambda=1 \mu \mathrm{m}$ and $1 / e$ halfdivergence angle $\theta_{\text {div }}=3 \mu \mathrm{rad}$, we obtain $L_{R} \equiv$ $\lambda /\left(\pi \theta_{\text {div }}^{2}\right)=35 \mathrm{~km}$. Equation (13) will enable us to view the wavefront of the beam as that of a spherical wave. However, the spherical-wave model is limited because the finite transverse extent of the beam has an influence on the coherence loss and imposes restrictions if a spherical-wave model is assumed. Because the WSF will be evaluated only for a spherical wave, we now enounce these restrictions. In the absence of atmospheric perturbations, let $W_{1}$ and $W$ be

Table 1. Geometrical Parameters Relevant for Links with LEO and/ or GEO Satellites

\begin{tabular}{lcc}
\hline & LEO & GEO \\
\hline Height $\left(H_{1}\right.$ or $\left.H_{2}\right)$ & $500 \mathrm{~km}$ & $36,000 \mathrm{~km}$ \\
Distance to perigee $\left(L_{1}\right.$ or $\left.L_{2}\right)$ & $2600 \mathrm{~km}$ & $42,000 \mathrm{~km}$ \\
\hline
\end{tabular}


the beam radii after the propagation distances $L_{1}$ and $L$, respectively. From Eq. (13), we have

$$
\theta_{\mathrm{div}} \approx \frac{W_{1}}{L_{1}} \approx \frac{W}{L}
$$

The relevant geometrical beam parameters are depicted in Fig. 4 . The incident angle $\theta$ of a wave mode reaching the receiver is limited by the finite size of the beam at the PS. So, the spherical-wave approximation can only be accurate if the incident angle is such that $\theta \ll W_{1} / L_{2}$. The remoteness of the receiver $(\mathrm{Rx})$ satellite from the PS acts as a spatial filter for the distortions of the received wave. The optical power lost because of this filtering corresponds to the beam-spread loss. The angle $\theta$ of the frequency domain is related to the separation distance $\rho$ of the spatial domain by the relation $\rho=1 /(\theta k)$, so that the restriction becomes $\rho \gg L_{2} /\left(k W_{1}\right)$. Additionally the spherical-wave WSF can only equal the Gaussian-beam WSF for spatial scales within the beam extent, that is, for $\rho \ll W$. We thus obtain the validity region

$$
\frac{1}{k} \frac{L_{2}}{W_{1}} \ll \rho \ll W
$$

By assuming a spherical wave, the WSF will be overestimated for $\rho<L_{2} /\left(k W_{1}\right)$. Appendix A shows how Eq. (15) can be derived from a relation previously derived by other authors. Using this relation [substantially reproduced in Eq. (65)], the error induced by the spherical-wave assumption can be estimated.

Because we assume statistically homogenous turbulence, distortions at the receiver should originate from a turbulent region with quasi-homogeneous parameters $C_{n}^{2}, S_{n}^{2}, L_{0, i}$, and $L_{0, a}$. In the vertical direction, we thus impose $\theta_{x} \ll H_{0} / L_{2}$, which in term of separation distance gives

$$
\frac{1}{k} \frac{L_{2}}{H_{0}} \ll \rho_{x}
$$

Considering an Rx satellite near the Earth (say $L_{2}<10^{5} \mathrm{~km}$ ), we can assume for the $y$ direction a statistically homogeneous refractive index. That is, we neglect refraction effects (turbulence or mean refraction) associated with the transverse limb curvature.

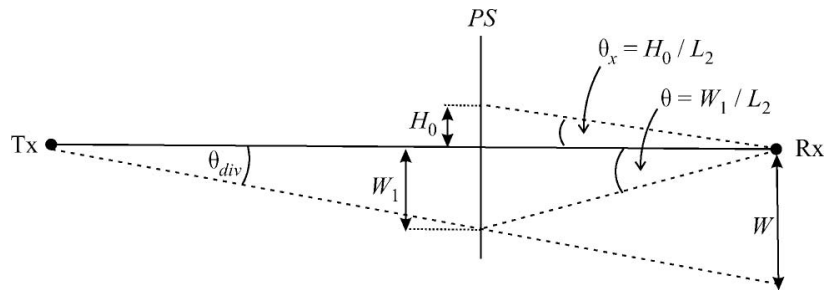

Fig. 4. Geometry of the considered beam model and angular relations (PS, phase screen).

\section{Mean Refraction}

Variation of the mean refractivity $\langle N(h)\rangle$ bends and defocuses the beam in the vertical direction. The total refraction angle, noted $\omega$, reaches $19 \mathrm{mrad}\left(1.1^{\circ}\right)$ when the perigee point is at the ground. It has therefore a negligible effect on the distances $L_{1}$ and $L_{2}$, and on the grazing angle $\alpha(z)$. However, the induced amount of beam spreading at the receiver may not be negligible and is determined by the vertical defocusing factor $\phi$ given by []ㅡ]

$$
\phi^{-1}=1-\frac{L_{1} L_{2}}{L} \frac{\mathrm{d} \omega}{\mathrm{d} h} .
$$

In writing Eq. (17), the PS approximation was used. Some authors interested in intensity measurements call $\phi$ the dilution factor [16].

\section{Spatial Wave Structure Function (Spherical Wave)}

Based on the Rytov theory, the transverse wave structure function $D_{w}(\rho)$ of a spherical wave (see Ref. [17], Subsection 6.4.2) can be written as

$$
D_{w}\left(\rho_{x}, \rho_{y}\right)=\int_{0}^{L} D_{w}^{z}\left(\rho_{x}, \rho_{y} ; z\right) \mathrm{d} z
$$

with

$$
\begin{array}{r}
D_{w}^{z}\left(\rho_{x}, \rho_{y} ; z\right)=4 \pi k^{2} \int_{-\infty}^{\infty} \int_{-\infty}^{\infty} \Phi_{n}\left(\kappa_{x}, \kappa_{y}, z\right) \\
{\left[1-e^{-j \xi\left(\phi \kappa_{x} \rho_{x}+\kappa_{y} \rho_{y}\right)}\right] \mathrm{d} \kappa_{x} \mathrm{~d} \kappa_{y},}
\end{array}
$$

where $\xi \equiv z / L$. Assuming $L_{\text {atm }} \ll L_{1}$ and $L_{\text {atm }} \ll L_{2}$, we apply the PS approximation in the exponential term of Eq. (19). The parameter $\phi$ is given by Eq. (17), and we set $\xi=L_{1} / L$. Next, we provide separate expressions for $D_{w, i}$ and $D_{w, a}$, the isotropic and anisotropic structure functions.

A. Isotropic Function

By changing the integration variables, Eq. (19) applied to isotropic turbulence becomes

$$
\begin{aligned}
D_{w, i}^{z}\left(\rho_{x}, \rho_{y} ; z\right)= & 8 \pi^{2} k^{2} \int_{0}^{\infty} \Phi_{n, i}\left(\kappa_{\perp} ; z\right)[1 \\
& \left.-J_{0}\left(\kappa_{\perp} r_{i}\right)\right] \kappa_{\perp} \mathrm{d} \kappa_{\perp}
\end{aligned}
$$

where $J_{0}$ is the Bessel function of the first kind and order zero, $\kappa_{\perp}^{2}=\kappa_{x}^{2}+\kappa_{y}^{2}$, and

$$
r_{i}=\frac{L_{1}}{L} \sqrt{\left(\phi \rho_{x}\right)^{2}+\rho_{y}^{2}} .
$$

The integration over $\kappa_{\perp}$ can be performed using formulas of Appendix B. The next step is to integrate Eq. (20) over $z$. Defining the width $\rho_{i, \text { atm }} \equiv$ $\left(1.46 k^{2} \mu_{0, i}\right)^{-3 / 5}$, we obtain the common results: 


$$
\begin{gathered}
D_{w, i}\left(\rho_{x}, \rho_{y}\right)=2\left(\frac{r_{i}}{\rho_{i, \text { atm }}}\right)^{5 / 3}, \quad r_{i} \ll L_{0, i}, \\
D_{w, i}\left(\rho_{x}, \rho_{y}\right)=0.05\left(\frac{L_{0, i}}{\rho_{i, \text { atm }}}\right)^{5 / 3}, \quad r_{i} \gg L_{0, i} .
\end{gathered}
$$

\section{B. Anisotropic Function}

Converting geocentric coordinates to beam coordinates, the evaluation of the anisotropic spectrum in the plane transverse to the beam yields

$$
\begin{aligned}
\Phi_{n, a}\left(\kappa_{x}, \kappa_{y}, z\right) & =S_{n}^{2}\left(\eta^{2} K_{X}^{2}+K_{Z}^{2}+\kappa_{0, a}^{2}\right)^{-5 / 2} \\
& =S_{n}^{2}\left(\eta^{2} \kappa_{y}^{2}+\gamma^{2} \kappa_{x}^{2}+\kappa_{0, a}^{2}\right)^{-5 / 2},
\end{aligned}
$$

with (see Ref. [15] for a detailed derivation)

$$
\gamma=\sqrt{\cos ^{2} \alpha+\eta^{2} \sin ^{2} \alpha}
$$

By changing the integration variables, Eq. (19) evaluated for the anisotropic part becomes

$$
D_{w, a}^{z}\left(\rho_{x}, \rho_{y} ; z\right)=8 \pi^{2} k^{2} S_{n}^{2}(\gamma \eta)^{-1} \int_{0}^{\infty} \frac{1-J_{0}\left(\kappa_{\perp} r_{a}\right)}{\left(\kappa_{\perp}^{2}+\kappa_{0, a}^{2}\right)^{5 / 2}} \kappa_{\perp} \mathrm{d} \kappa_{\perp},
$$

where

$$
r_{a}=\frac{L_{1}}{L} \sqrt{\left(\phi \gamma^{-1} \rho_{x}\right)^{2}+\left(\eta^{-1} \rho_{y}\right)^{2}} .
$$

As for the isotropic function, Eq. (26) can be integrated using Appendix $\mathrm{A}$. When integrating over $z$, an obstacle resides in the fact that $\alpha$ and hence $\gamma$ depend on $z$. Defining

$$
\mu_{m, a} \equiv \int_{0}^{L} S_{n}^{2} \gamma^{-m} \mathrm{~d} z
$$

we must deal with $\mu_{1, a}$ in the $x$ direction, and with $\mu_{3, a}$ in the $y$ direction. In the stratospheric region, we find for $\eta=30$ that $\mu_{1, a}=0.86 \mu_{0, a}$ and $\mu_{3, a}=0.69 \mu_{0, a}$. To simplify expressions, we make the approximation $\gamma \approx$ 1 and thus $\mu_{3, a} \approx \mu_{1, a} \approx \mu_{0, a}$. This allows us to define $\rho_{a, \text { atm }} \equiv\left(1 / 4 \pi k^{2} L_{0, a} \mu_{0, a} \eta^{-1}\right)^{-1 / 2}$ and to obtain the two asymptotic results:

$$
\begin{gathered}
D_{w, a}\left(\rho_{x}, \rho_{y}\right)=2\left(\frac{r_{a}}{\rho_{a, \text { atm }}}\right)^{2}, \quad r_{a} \ll L_{0, a}, \\
D_{w, a}\left(\rho_{x}, \rho_{y}\right)=\frac{1}{6 \pi^{2}}\left(\frac{L_{0, a}}{\rho_{a, \text { atm }}}\right)^{2}, \quad r_{a} \gg L_{0, a} .
\end{gathered}
$$

Note that Gurvich and Belen'kii derived a quadratic expression similar to Eq. (29) for stellar observation from the ground [10].

\section{Coherence Width}

The total WSF is the sum of the isotropic and anisotropic parts. The coherence widths $\rho_{0, x}$ and $\rho_{0, y}$ in the $x$ and $y$ directions, respectively, are defined as the separation distances for which the structure function equals 2:

$$
D_{w}\left(\rho_{0, x}, 0\right) \equiv D_{w}\left(0, \rho_{0, y}\right) \equiv 2 .
$$

We wish to separate the influence of the atmosphere and the influence of the satellite positions. To this aim, we consider $\rho_{0, \operatorname{atm} x x}$ and $\rho_{0, \operatorname{atm}, y}$ the coherence widths of a wave field located just after the PS, that is with $L_{2}=0$ :

$$
\left.\left.D_{w}\left(\rho_{0, \operatorname{atm}, x}, 0\right)\right|_{L_{2}=0} \equiv D_{w}\left(0, \rho_{0, \operatorname{atm}, y}\right)\right|_{L_{2}=0} \equiv 2 .
$$

Setting $L_{2}=0$ leads to $L_{1}=L$ and $\phi=1$, so that we can write

$$
\left[\begin{array}{l}
\rho_{0, x} \\
\rho_{0, y}
\end{array}\right] \approx \frac{L}{L_{1}}\left[\begin{array}{l}
\phi^{-1} \rho_{0, \mathrm{~atm}, x} \\
\rho_{0, \mathrm{~atm}, y}
\end{array}\right] .
$$

The equality in Eq. (33) is only approximate because $D_{w, i}$ and $D_{w, a}$ do not have the same power law for their argument. Equation (33) is thus slightly inac-

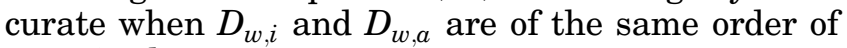
magnitude.

In Fig. 5, the influence of satellite positions is examined, and the expansion factor $\phi^{-1} L / L_{1}$ is plotted for the four different scenarios involving LEO and GEO satellites. One sees that, in terms of wavefront distortions at the receiver, the GEO-to-LEO link, which is close to the plane-wave scenario, corresponds to the worst case. Figure 6 shows the

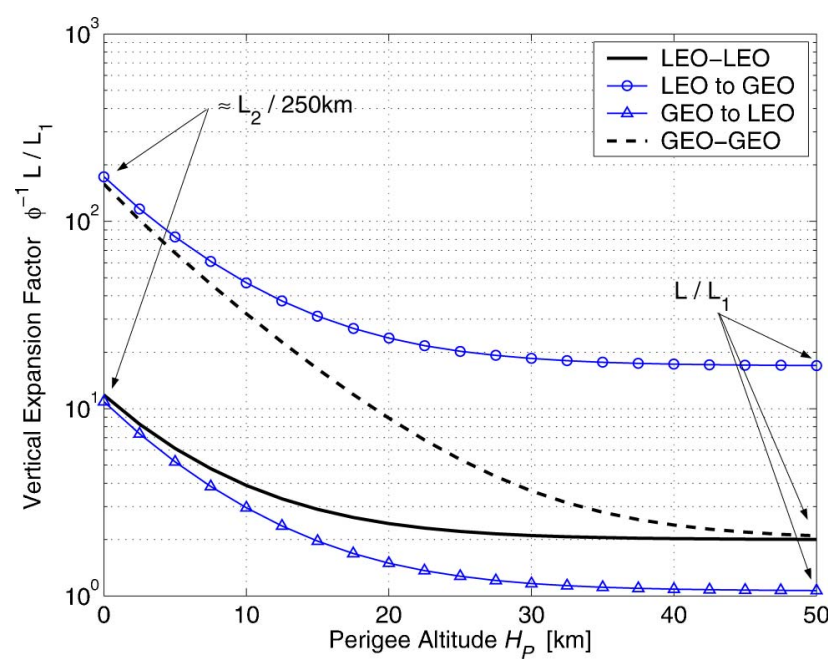

Fig. 5. (Color online) Vertical expansion factor $\phi^{-1} L / L_{1}$ for four different links. 


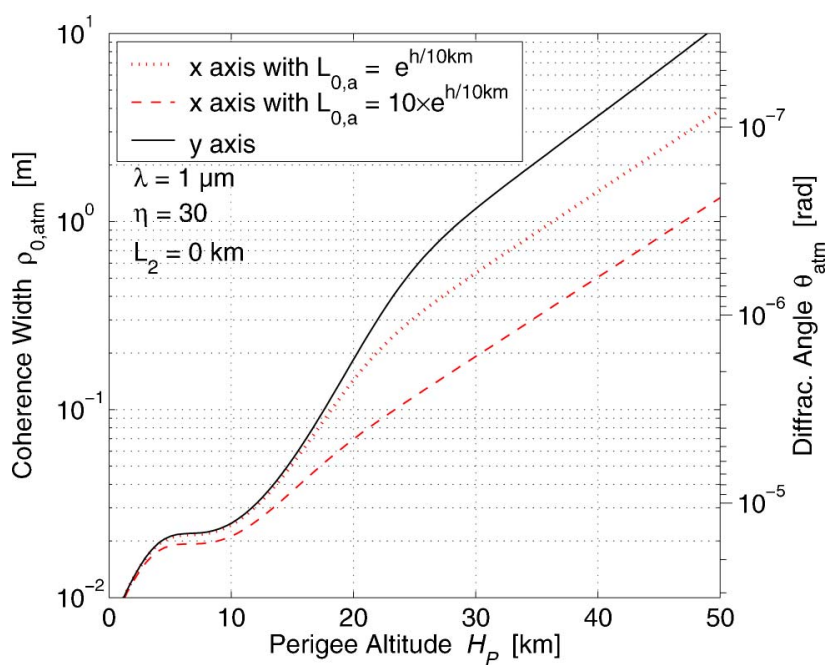

Fig. 6. (Color online) Normalized coherence widths $\rho_{0, a t m, x}$ and $\rho_{0, \text { atm } y}$ defined with $L_{2}=0$ and plotted for $\lambda=1 \mu \mathrm{m}$. The corresponding values for the diffraction angles $\theta_{\mathrm{atm}, x}$ and $\theta_{\mathrm{atm}, y}$ are displayed on the right axis.

coherence widths $\rho_{0, \text { atm } x}$ and $\rho_{0, \text { atm } y}$ as a function of the perigee altitude for $\lambda=1 \mu \mathrm{m}$. Two different profiles of $L_{0, a}$ are considered: $L_{0, a}(h)=e^{h / 10 \mathrm{~km}}$ and $L_{0 . a}(h)=10 \times e^{h / 10 \mathrm{~km}}$. We note that for any altitude $H_{P}$, the widths $\rho_{0, \text { atm } x}$ and $\rho_{0, \text { atm } y}$ are much smaller than typical values of $L_{0, i}$ or $L_{0, a}$ and thus could be calculated by considering Eqs. (22) and (29). The right ordinate axis of Fig. 6 shows the values taken by the diffraction angle $\theta_{\text {atm }}$ associated with the loss of coherence. This angle is defined for both transverse components as

$$
\left[\begin{array}{c}
\theta_{\operatorname{atm}, x} \\
\theta_{\operatorname{atm}, y}
\end{array}\right] \equiv \frac{2}{k}\left[\begin{array}{l}
1 / \rho_{0, \operatorname{atm}, x} \\
1 / \rho_{0, \text { atm }, y}
\end{array}\right]
$$

For both considered outer-scale profiles, we found that the coherence width in the $y$ direction is dominated by the isotropic turbulence so that we can write

$$
D_{w}\left(0, \rho_{y}\right) \approx D_{w, i}\left(0, \rho_{y}\right)
$$

In the upper atmosphere $\left(H_{P}>25 \mathrm{~km}\right)$, both coherence widths $\rho_{0, \text { atm } x}$ and $\rho_{0, \text { atm } y}$ increase with $H_{P}$ at an exponential rate with a characteristic height of $\sim 6 \mathrm{~km}$. Figures $\underline{5}$ and $\underline{6}$ reveal that in the $x$ direction two different phenomena act within quasi-separate altitude regions. Below $\sim 20 \mathrm{~km}$, mean-refraction defocusing noticeably enhances the vertical coherence, whereas above $\sim 20 \mathrm{~km}$ anisotropic irregularities make $\rho_{0, x}$ significantly smaller than $\rho_{0, y}$.

The Gaussian-beam conditions of Eq. (15) can be applied to the atmospheric quantities $\bar{\rho}_{0, \text { atm }}$ and $\theta_{\text {atm }}$. The upper bound on $\rho$ yields $\rho_{0 \text { atm }} \ll W_{1}$ in which we ignore the $\phi$ factor. The lower bound on $\rho$ is most restrictive for $L_{1} \ll L_{2}$ (e.g., a LEO-toGEO link), which yields simply $\theta_{\text {atm }} \ll \theta_{\text {div }}$. The homogeneous-turbulence condition of Eq. (16) yields $\theta_{\text {atm } x} \ll H_{0} L /\left(\phi L_{1} L_{2}\right)$. One can reasonably assume that this last condition is always fulfilled.

\section{Angle of Arrival}

The aperture diameter $D$ of the receiver is typically much smaller than the outer scales. Furthermore, under the geometric-optics approximation, the wave structure function $D_{w}$ is equal to the phase structure function. In this context, the variance $\sigma_{\mathrm{AoA}}^{2}$ of the angle of arrival (AoA) can be derived from what we call the "two-point tilt" approximation according to (see Ref. [17], Subsection 6.5):

$$
\left[\begin{array}{l}
\sigma_{\mathrm{AoA}, x}^{2} \\
\sigma_{\mathrm{AoA}, y}^{2}
\end{array}\right]=\frac{1}{k^{2} D^{2}}\left[\begin{array}{l}
D_{w}(D, 0) \\
D_{w}(0, D)
\end{array}\right]
$$

The two-point tilt approach amounts to defining the tilt in a given direction as the straight line that crosses the two diametrically opposed phase points on the aperture edge. Rigorously, this simplified two-point tilt is different from the average tilt over the whole aperture. However, if we, for example, consider the Kolmogorov spectrum, the apertureaveraged tilt variance as derived by Fried in [18] or as derived by Cheon and Muschinski within the geometric-optics approximation in Ref. [19] is close (error $<3 \%)$ to that derived from Eq. $(3 \overline{6})$.

Now, to reach conveniently an estimation of $\sigma_{\mathrm{AoA}, x}$ and $\sigma_{\mathrm{AoA} y}$, we assume the structure function takes the quadratic form $D_{w}=2\left(\rho_{x}^{2} / \rho_{0, x}^{2}+\rho_{y}^{2} / \rho_{0, y}^{2}\right)$. Equation (36) then yields

$$
\left[\begin{array}{l}
\sigma_{\mathrm{AoA}, x} \\
\sigma_{\mathrm{AoA}, y}
\end{array}\right] \approx \frac{\sqrt{2}}{k}\left[\begin{array}{l}
1 / \rho_{0, x} \\
1 / \rho_{0, y}
\end{array}\right]
$$

According to Eq. (36), the angle of arrival is independent of the wavelength but depends slightly on $D$ through the function $D_{w, i}$, which follows a 5/3-power law. In the approximation of Eq. (37), the dependence on $D$ is suppressed, but a small dependence on $\lambda$ is fictively introduced. Using Eq. (34) and (37),

$$
\left[\begin{array}{c}
\sigma_{\mathrm{AoA}, x} \\
\sigma_{\mathrm{AoA}, y}
\end{array}\right] \approx \frac{1}{\sqrt{2}} \frac{L_{1}}{L}\left[\begin{array}{c}
\phi \theta_{\mathrm{atm}, x} \\
\theta_{\mathrm{atm}, y}
\end{array}\right] .
$$

Figure 7 shows the pointing errors measured by the OICETS satellite over a GEO-to-LEO link [9]. In the upper graph the angle in the vertical or $x$ direction is plotted, whereas the angle in the horizontal or $y$ direction is plotted in the lower graph [20]. Although one can observe that the fluctuation strength is similar in orders of magnitude to those deduced from Eq. (38), one cannot directly compare those data with our theoretical results. This is because the opticaltracking system on the satellite tends to compensate for angular deviations and also may have been perturbed by scintillation. Additionally, some time averaging may have taken place when detecting the angle of arrival (the sampling rate of the sensors was $4 \mathrm{kHz}$ ). 

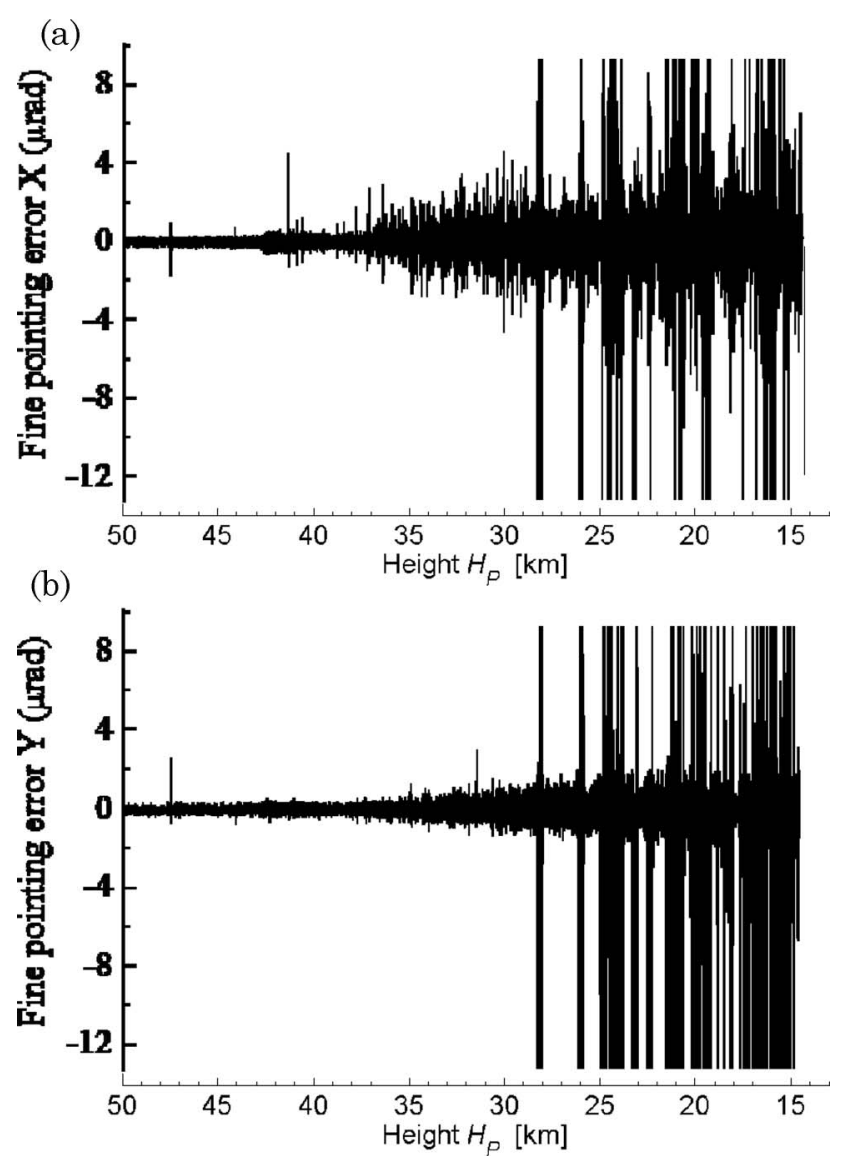

Fig. 7. Fluctuations of the AoA as recorded by the OICETS satellite during a link with the ARTEMIS satellite. The upper graph shows the vertical angular component and the lower graph shows the horizontal one (courtesy of Takayama et al.. [9]]).

\section{Temporal Wave Structure Function (Spherical Wave)}

\section{A. General Relations}

To derive the temporal statistics of turbulence-induced fluctuations, we rely on the common frozenturbulence hypothesis [21]. The speed at which turbulent cells cross the optical beam is essentially determined by the beam motion driven by the satellite speeds (atmospheric wind is neglected). Let $\mathbf{V}_{1, \perp}$ and $\mathbf{V}_{2, \perp}$ be the transverse speed vectors of, respectively, the transmitting and receiving satellites as observed from the Earth center. Let $\mathbf{V}_{\mathrm{RW}, 0}$ be the transverse relative-wind speed vector when mean refraction is hypothetically uniformly zero. Along the beam path, $\mathbf{V}_{\mathrm{RW}, 0}$ is given by

$$
\mathbf{V}_{\mathrm{RW}, 0}(z)=(1-\xi) \mathbf{V}_{1, \perp}+\xi \mathbf{V}_{2, \perp}
$$

We note $\mathbf{V}_{\mathrm{RW}}$ the transverse relative-wind speed vector including vertical mean refraction. The components of $\mathbf{V}_{\mathrm{RW}}$ in the atmospheric range of $z$ are then

$$
\left[\begin{array}{c}
V_{\mathrm{RW}, x} \\
V_{\mathrm{RW}, y}
\end{array}\right]=\left[\begin{array}{c}
\phi V_{\mathrm{RW}, 0, x} \\
V_{\mathrm{RW}, 0, y}
\end{array}\right], \quad\left|z-L_{1}\right|<L_{\mathrm{atm}} .
$$

In writing Eq. (40), we have made two approximations about transverse speeds. The first approximation is related to the PS approximation since $\phi V_{\mathrm{RW}, 0, x}$ represents the vertical velocity of the bending point on the PS and not the actual vertical velocity of the perigee point. This issue was addressed by Dalaudier et al. who showed that the ratio of the two velocities is close to one (Subsection 4.C of Ref. [16]). The second approximation consists of neglecting the effect of mean refraction on the transverse speeds $\mathbf{V}_{1, \perp}$ and $\mathbf{V}_{2 \perp}$ because the associated angles are small.

Now, taking defocusing effects into account, the equivalence between spatial and temporal arguments is

$$
\left[\begin{array}{l}
V_{\mathrm{RW}, x} \\
V_{\mathrm{RW}, y}
\end{array}\right] \tau=\xi\left[\begin{array}{c}
\phi \rho_{x} \\
\rho_{y}
\end{array}\right] .
$$

Introducing Eq. (41) into Eqs. (20) and (26) we obtain, for an outer scale large enough,

$$
\begin{gathered}
D_{w, i}^{z}(\tau ; z)=2.9 k^{2} C_{n}^{2}\left(V_{\mathrm{RW}} \tau\right)^{5 / 3} \\
D_{w, a}^{z}(\tau ; z)=1 / 2 \pi k^{2} L_{0, a} S_{n}^{2}(\gamma \eta)^{-1}\left(\gamma^{-2} V_{\mathrm{RW}, x}^{2}\right. \\
\left.+\eta^{-2} V_{\mathrm{RW}, y}^{2}\right) \tau^{2}
\end{gathered}
$$

Integrating over $z$, we use again $\mu_{3, a} \approx \mu_{1, a} \approx \mu_{0, a}$ for the anisotropic part and write

$$
D_{w, i}(\tau)=2\left(\frac{V_{\mathrm{eq}, i} \tau}{\rho_{i, \mathrm{~atm}}}\right)^{5 / 3}
$$

$$
D_{w, a}(\tau)=2\left(\frac{\sqrt{V_{\mathrm{eq}, a, x}^{2}+\eta^{-2} V_{\mathrm{eq}, a, y}^{2} \tau}}{\rho_{a, \mathrm{~atm}}}\right)^{2},
$$

with

$$
\begin{gathered}
V_{\mathrm{eq}, i}=\left(\mu_{0, i}^{-1} \int_{0}^{L} C_{n}^{2} V_{\mathrm{RW}}^{5 / 3} \mathrm{~d} z\right)^{3 / 5}, \\
V_{\mathrm{eq}, a, x}=\left(\mu_{3, a}^{-1} \int_{0}^{L} S_{n}^{2} \gamma^{-3} V_{\mathrm{RW}, x}^{2} \mathrm{~d} z\right)^{1 / 2}, \\
V_{\mathrm{eq}, a, y}=\left(\mu_{1, a}^{-1} \int_{0}^{L} S_{n}^{2} \gamma^{-1} V_{\mathrm{RW}, y}^{2} \mathrm{~d} z\right)^{1 / 2} .
\end{gathered}
$$

Similarly to the coherence width, we define the coherence time $\tau_{0}$ by $D_{w}\left(\tau_{0}\right) \equiv 2$, and we obtain the following limit cases: 


$$
\tau_{0}=\left\{\begin{array}{ll}
\frac{\rho_{0, \mathrm{~atm}}}{V_{\mathrm{eq}, i}} & \text { if } D_{S, a}(\tau) \ll D_{S, i}(\tau) \\
\left(\frac{V_{\mathrm{eq}, a, x}}{\rho_{0, a \mathrm{tm}, x}^{2}}+\frac{V_{\mathrm{eq}, a, y}^{2}}{\rho_{0, \mathrm{~atm}, y}^{2}}\right)^{-1 / 2} & \text { if } D_{S, i}(\tau) \ll D_{S, a}(\tau)
\end{array} .\right.
$$

In the following, the fluctuation speeds of two different scenarios are evaluated for illustration purposes. For both examples, fluctuation speed is independent of the link direction.

\section{B. "LEO Chasing LEO" Link}

In a "LEO chasing LEO" scenario, both LEO satellites have the same trajectory in a geocentric frame of reference, and one satellite is delayed with respect to the other satellite. It results that $H_{P}$ is constant over time and that the satellite speed vectors follow the relations

$$
\begin{aligned}
& \mathbf{V}_{1, \perp}=V_{1}\left[\begin{array}{c}
L_{1} /\left(R_{e}+H_{1}\right) \\
0
\end{array}\right] . \\
& \mathbf{V}_{2, \perp}=-\mathbf{V}_{1, \perp}
\end{aligned}
$$

In that case, Eqs. (39) and (0ㅡ) yield

$$
\left[\begin{array}{l}
V_{\mathrm{RW}, x} \\
V_{\mathrm{RW}, y}
\end{array}\right]=\left[\begin{array}{c}
\phi(1-2 \xi) V_{1, x} \\
0
\end{array}\right] \text {. }
$$

The transverse relative wind is displayed in Fig. 8 . The coherence time $\tau_{0}$ is plotted over $H_{P}$ in Fig. $\overline{9}$ (upper plot). The altitude of the LEO satellites corresponds to Table $\underline{1}$, and the speed $V_{1}$ of the satellites is $7.5 \mathrm{~km} / \mathrm{s}$. Because the beam moves vertically through the atmosphere, anisotropic irregularities mostly determine the coherence time in the upper atmosphere. This is noticeable through the consideration of two different outer-scale profiles $\left(L_{0, a}(h)=e^{h / 10 \mathrm{~km}}\right.$ and $\left.10 \times e^{h / 10 \mathrm{~km}}\right)$.

\section{LEO-GEO Link}

Let us consider a LEO-GEO link. Let $\mathbf{V}_{\mathrm{LEO}, \perp}$ (respectively $\mathbf{V}_{\mathrm{GEO}, \perp}$ ) be the transverse speed of the LEO (respectively GEO) satellite and $L_{\mathrm{LEO}}$ (respectively $L_{\mathrm{GEO}}$ ) be the distance between the perigee point and the LEO (respectively GEO) satellite. Because $\mathbf{V}_{\mathrm{GEO}, \perp}=0$ and $L_{\mathrm{GEO}} \gg L_{\mathrm{LEO}}$, the GEO terminal falls into the category of remote slow terminals with respect to the LEO terminal. This implies that, within the atmospheric region, Eq. (39) becomes $\mathbf{V}_{\mathrm{RW}, 0} \approx \mathbf{V}_{\mathrm{LEO}, \perp}$. It also implies that $\bar{\phi}^{-1} \approx \phi_{\mathrm{LEO}}^{-1} \equiv$

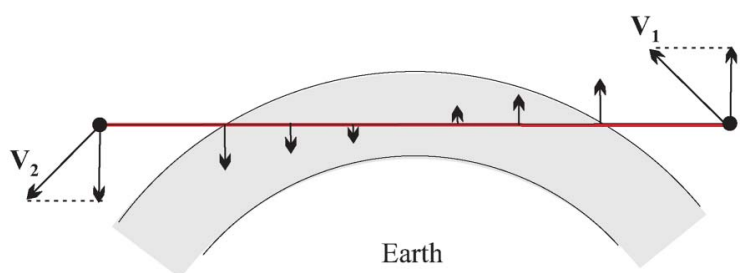

Fig. 8. (Color online) Transverse component of the relative wind for a "LEO-chasing-LEO" link.

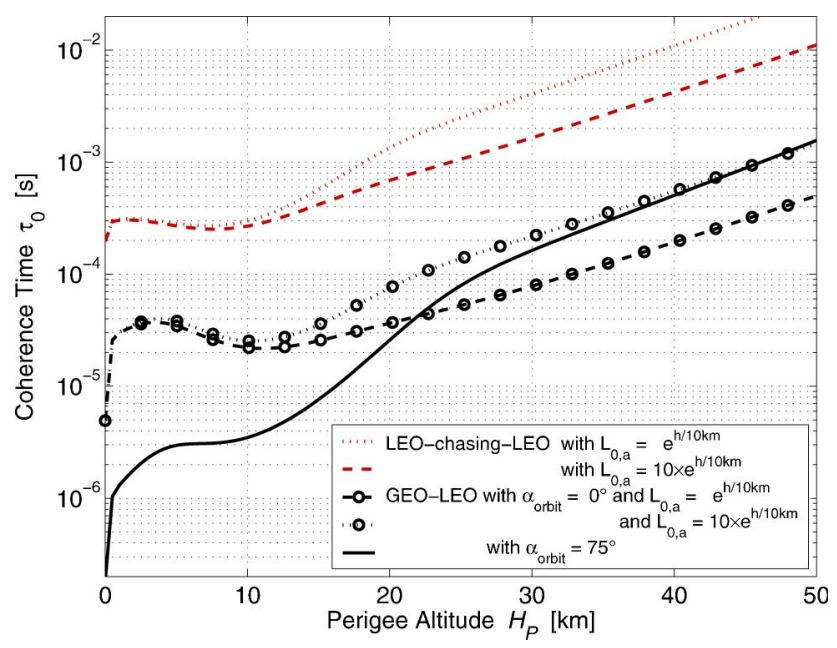

Fig. 9. (Color online) Coherence time $\tau_{0}$ calculated as a function of $H_{P}$ for various scenarios.

$1-L_{\mathrm{LEO}} \mathrm{d} \omega / \mathrm{d} h$, so that for values of $z$ in the atmosphere we have

$$
\left[\begin{array}{c}
V_{\mathrm{RW}, x} \\
V_{\mathrm{RW}, y}
\end{array}\right] \approx\left[\begin{array}{c}
\phi_{\mathrm{LEO}} V_{\mathrm{LEO}, x} \\
V_{\mathrm{LEO}, y}
\end{array}\right] .
$$

Because $\mathbf{V}_{\mathrm{RW}}$ is independent of $z$, the coherence time $\tau_{0}$ is the same for both link directions. $\mathbf{V}_{\mathrm{LEO}, \perp}$ has a negligible dependence on $H_{P}$ for $H_{P}<50 \mathrm{~km}$. However, $\mathbf{V}_{\mathrm{LEO}, \perp}$ has a strong dependence on the orbit inclination $\alpha_{\text {orbit }}$ with respect to the Earth-GEO axis. Figure 10(a) depicts a LEO orbit and indicates the inclination parameter $\alpha_{\text {orbit }}$. Figure $10(\mathrm{~b})$ depicts the orbit of the LEO satellite as seen from the GEO satellite for two $\alpha_{\text {orbit }}$ values. The components of $\mathbf{V}_{\text {LEO } \perp \perp}$ can be calculated through projection relations. The components of $\mathbf{V}_{\mathrm{RW}}$ are plotted in Fig. 11 as a function of $\alpha_{\text {orbit }}$.

Along with the previous example, Fig. 9 shows the coherence time of a LEO-GEO link for $\alpha_{\text {orbit }}=0^{\circ}$ and $75^{\circ}$. For the case $\alpha_{\text {orbit }}=0^{\circ}$, the beam moves vertically through the atmosphere, and two different outer-scale profiles $\left(L_{0, a}(h)=e^{h / 10 \mathrm{~km}}\right.$ and $\left.10 \times e^{h / 10 \mathrm{~km}}\right)$ have been considered. One sees that a LEO-GEO link presents faster fluctuations than the "LEO chasing LEO” link.

\section{Beam Spread and Beam Wander}

Beam spread and beam wander increase as the beam radius decreases; therefore the spherical-wave approximation cannot be applied anymore. Analyses of Subsections 6.A and 6.B are based on the assumption that the turbulence strength is the same across the whole beam cross section. To fulfill this condition, one may reasonably consider $W_{1}<500 \mathrm{~m}$. This restriction on $W_{1}$ is equivalent to $\theta_{\text {div }}<190 \mu \mathrm{rad}$ for a transmitting LEO satellite and $\theta_{\text {div }}<12 \mu \mathrm{rad}$ for a transmitting GEO satellite. This restriction will not affect the scope of the results much because, for $W_{1}=500 \mathrm{~m}$, turbulence-induced beam spread and beam wander are already very weak. 


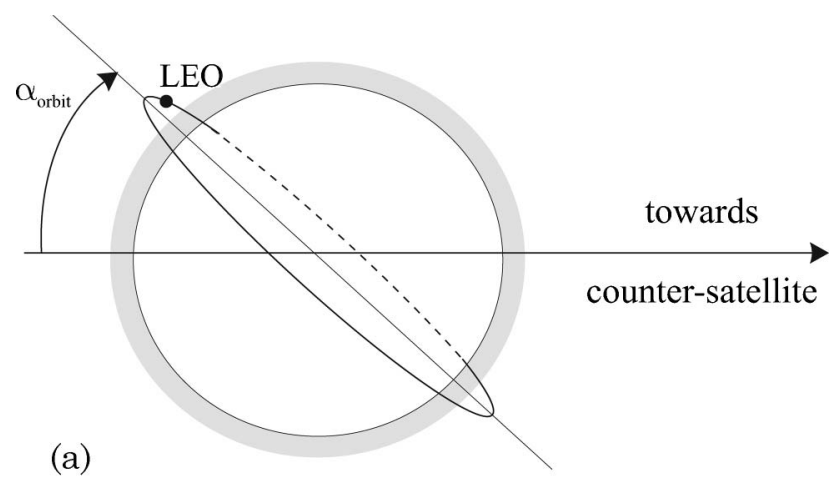

(b)

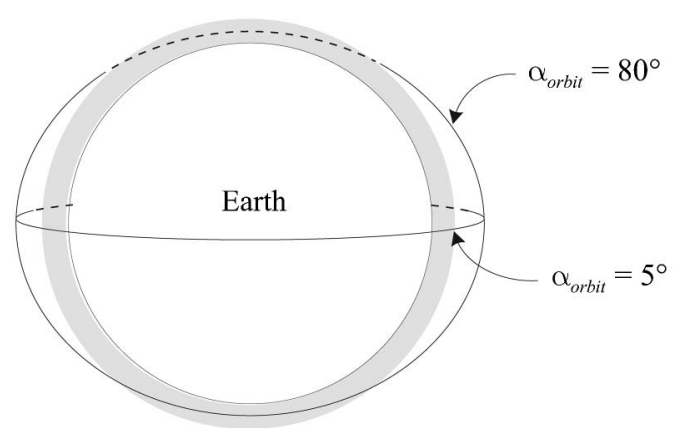

Fig. 10. Geometry of an occultation link with a LEO satellite and a remote countersatellite: (a) the LEO orbit makes an angle $\alpha_{\text {orbit }}$ with respect to the direction toward the countersatellite, (b) the LEO orbit is traced as seen from the countersatellite.

\section{A. Beam Spread}

The beam spread is found by calculating the mean intensity profile at the receiver. To this end, we apply the extended Huygens-Fresnel principle $[17,22]$ for the propagation of the beam from the PS to the receiver. We separate the mean-refraction effect from the turbulence effect by considering an initial beam located at $z=L_{1}$ and already affected by the vertical defocusing factor $\phi$ but not affected yet by the turbulent PS located at $z=L_{1}^{+}$. The initial beam is thus

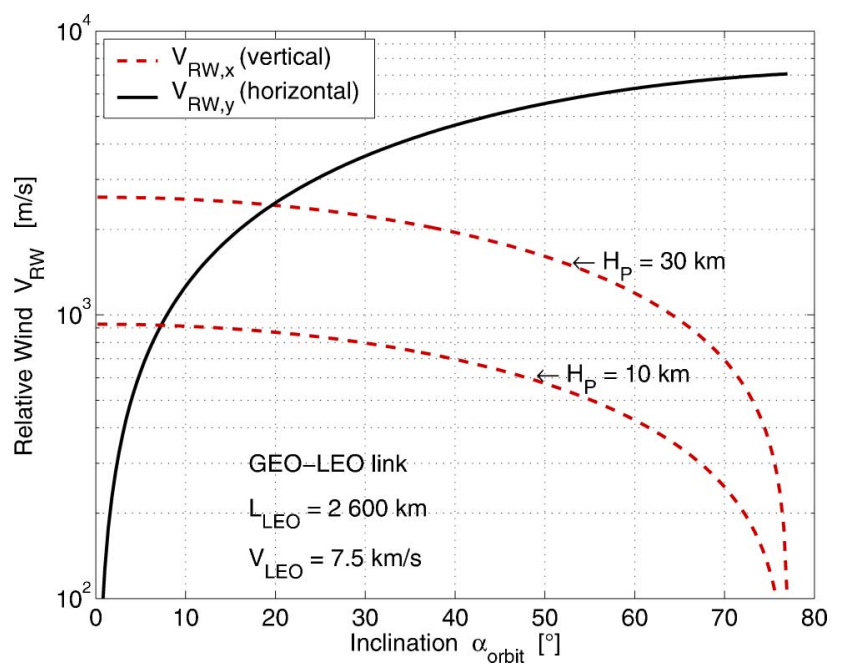

Fig. 11. (Color online) Relative transverse wind $V_{\mathrm{RW}}$ as a function of the LEO orbit inclination $\alpha_{\text {orbit }}$ in the case of a LEOGEO link. modeled as an astigmatic Gaussian beam with a vertical curvature radius reduced by the factor $\phi$. The extended Huygens-Fresnel principle has already been applied to astigmatic Gaussian beams by other authors [23]. The intensity profile $\langle I(x, y)\rangle$ at $z=L$ normalized to have an on-axis value of 1 without atmospheric effects is then given by

$$
\begin{aligned}
\langle I(x, y)\rangle= & \frac{k^{2} W^{2}}{8 \pi L_{2}^{2}} \iint_{-\infty}^{\infty} \mathrm{d} Q_{x} \mathrm{~d} Q_{y} \exp \left[-i \frac{k}{L_{2}}\left(x Q_{x}+y Q_{y}\right)\right] \\
& \times \exp \left[-\frac{k^{2} W^{2}}{8 L_{2}^{2}}\left(\frac{Q_{x}^{2}}{\phi^{2}}+Q_{y}^{2}\right)\right] \\
& \times \exp \left[-\frac{1}{2} D_{\mathrm{sp}}\left(Q_{x}, Q_{y}\right)\right] .
\end{aligned}
$$

$D_{\mathrm{sp}}\left(Q_{x}, Q_{y}\right)$ is the phase structure function of a spherical wave propagating from the receiver to just behind the PS (at $z=L_{1}^{-}$). We consider the simple form

$$
D_{\mathrm{sp}}\left(Q_{x}, Q_{y}\right)=2\left(\frac{Q_{x}^{2}}{\rho_{0, \mathrm{~atm}, x}^{2}}+\frac{Q_{y}^{2}}{\rho_{0, \mathrm{~atm}, y}^{2}}\right) .
$$

Equation (53) amounts then to the Fourier transform of a Gaussian function, which is another Gaussian function. We find

$$
\langle I(x, y)\rangle=\frac{1}{s_{x} s_{y}} \exp \left[-2 \frac{x^{2}}{s_{x}^{2} W^{2}}-2 \frac{y^{2}}{s_{y}^{2} W^{2}}\right],
$$

where, using Eqs. (34) and (14), the spread factors $s_{x}$ and $s_{y}$ are given by

$$
s_{x}^{2}=\phi^{-2}+2\left(\frac{L_{2}}{L} \frac{\theta_{\mathrm{atm}, x}}{\theta_{\mathrm{div}}}\right)^{2},
$$

$$
s_{y}^{2}=1+2\left(\frac{L_{2}}{L} \frac{\theta_{\mathrm{atm}, y}}{\theta_{\mathrm{div}}}\right)^{2} .
$$

Figure 12 shows the beam-spread loss $1 /\left(s_{x} s_{y}\right)$ for a beam originating from a LEO satellite with $\lambda=1 \mu \mathrm{m}$. Two divergence angles ( 3 and $30 \mu \mathrm{rad}$ ) and two types of receiver satellite (LEO and GEO) are considered. Because beam spread is weak at altitudes with significant anisotropic irregularities, $L_{0, a}$ has a limited effect on the beam spread. Figure 12 shows the case $L_{0, a}=5 \times e^{h / 10 \mathrm{~km}}$, and modifying $L_{0, a}$ makes the curves change slightly for $H_{P}>20 \mathrm{~km}$. By increasing $\theta_{\text {div }}$ from 3 to $30 \mu \mathrm{rad}$, most of turbulence-induced beam spread is removed, and the effect of the defocusing factor $\phi$ becomes discernible.

\section{B. Beam Wander}

Beam wander is highly dependent on the outer scale whether turbulence is isotropic or anisotropic. To keep the analysis simple, we consider the same 


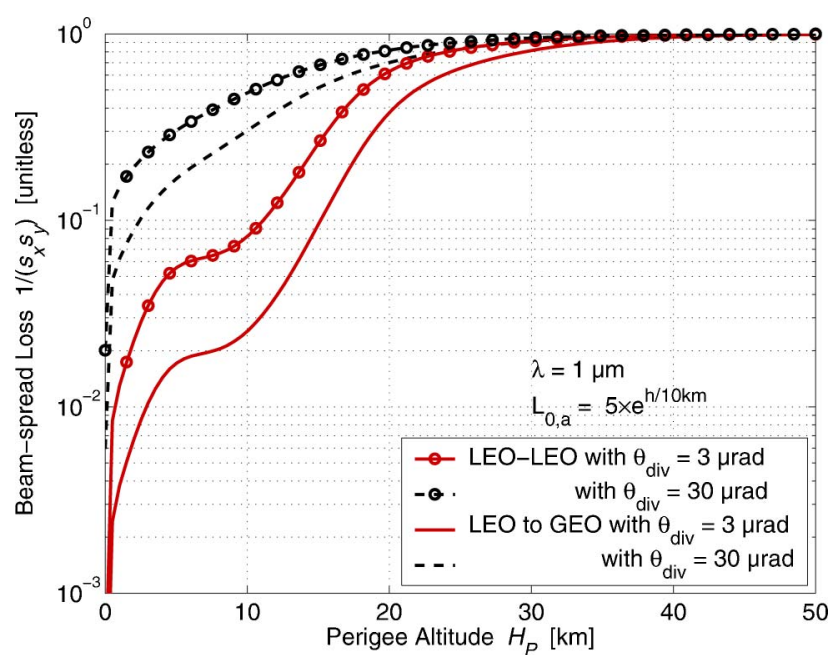

Fig. 12. (Color online) Beam-spread loss as a function of perigee altitude for $\lambda=1 \mu \mathrm{m}$.

atmospheric profile for isotropic and anisotropic outer scales and set $L_{0, i}=L_{0, a}=L_{0}$.

\section{Absolute Deviations}

We note $\sigma_{\mathrm{BW}}^{2}$ is the variance of the angular beam deviations caused by the atmospheric PS. As in Subsection 4.D, we rely on geometric optics and apply the two-point tilt approximation. Before commenting on the accuracy of the approach, we write the associated expression for the Gaussian beam located at the PS:

$$
\left[\begin{array}{c}
\sigma_{\mathrm{BW}, x}^{2} \\
\sigma_{\mathrm{BW}, y}^{2}
\end{array}\right]=\left.\frac{1}{4 k^{2} W_{1}^{2}}\left[\begin{array}{c}
D_{w}\left(2 W_{1}, 0\right) \\
D_{w}\left(0,2 W_{1}\right)
\end{array}\right]\right|_{L_{2}=0},
$$

where $D_{w}$ is evaluated with $L_{2}=0$. As the ratio $W_{1} / L_{0}$ becomes significant, the two-point tilt approximation worsens because more aperture averaging of the tilt takes place. The approach is thus conservative, as the tilt tends to be overestimated. Considering for example the isotropic von Kármán spectrum given by Eq. (2), an analysis of the aperture-averaged tilt [see Eqs. (8) and (9) of Ref. [24]] leads to $\sigma_{\mathrm{BW}}^{2} \propto$ $W_{1}^{-1 / 3}\left(W_{1} / L_{0}\right)^{-11 / 3}$ for $W_{1} \gg L_{0}$, whereas the twopoint tilt based on Eqs. (58) and (23) leads to $\sigma_{\mathrm{BW}}^{2} \propto W_{1}^{-1 / 3}\left(W_{1} / L_{0}\right)^{-5 / 3}$. Note also that Andrews et al., who estimated the aperture-averaged beam wander with the 11/3-power spectrum including an outer scale but not with a von Kármán spectrum, derived an expression (see Eq. (118) of Ref. [17], p. 210) yielding $\sigma_{\mathrm{BW}}^{2} \propto W_{1}^{-1 / 3}\left(W_{1} / L_{0}\right)^{-5 / 3}$ for $W_{1} \gg L_{0}$.

Focusing on Eq. (58), we use our previous WSF relations together with Eq. (34) to derive the following asymptotic expressions:

$$
\left[\begin{array}{c}
\sigma_{\mathrm{BW}, x} \\
\sigma_{\mathrm{BW}, y}
\end{array}\right] \approx \frac{1}{\sqrt{2}}\left[\begin{array}{c}
\theta_{\mathrm{atm}, x} \\
\theta_{\mathrm{atm}, y}
\end{array}\right], \quad W_{1} \ll L_{0}
$$

$$
\left[\begin{array}{c}
\sigma_{\mathrm{BW}, x} \\
\sigma_{\mathrm{BW}, y}
\end{array}\right] \approx c \frac{L_{0}}{W_{1}}\left[\begin{array}{l}
\theta_{\mathrm{atm}, x} \\
\theta_{\mathrm{atm}, y}
\end{array}\right], \quad W_{1} \gg L_{0}
$$

where $c=0.056$ if the isotropic spectrum dominates or $c=0.032$ if the anisotropic spectrum dominates. Note that in Eqs. (59) and (60) we have once more approximated the $\overline{5 / 3}$-power law of the isotropic spectrum by a square law.

\section{Relative Deviations}

It is of interest to compare the strength of the beam deviations with the actual extent of the beam. Having a long-term Gaussian beam of radius $W_{\mathrm{LT}}$ and Gaussian-distributed beam displacements of standard deviation $\sigma_{c}$, we are led to a short-term Gaussian beam defined by its radius $W_{\mathrm{ST}}$. The relation between the three quantities is (see Eq. (13) of Ref. [25])

$$
W_{\mathrm{LT}, x}^{2}=W_{\mathrm{ST}, x}^{2}+2 \sigma_{c, x}^{2},
$$

in which only the $x$ component has been considered. In Eq. (61) and in the subsequent equations, replacing $x$ by $y$ and setting $\phi=1$ lead to the relations for the $y$ component. We define the relative beam wander by

$$
\beta_{x}^{2} \equiv \frac{2 \sigma_{c, x}^{2}}{W_{\mathrm{ST}, x}^{2}}=\frac{1}{W_{\mathrm{LT}, x}^{2} /\left(2 \sigma_{c, x}^{2}\right)-1} .
$$

Having the relations $W_{\mathrm{LT} . x}=s_{x} L \theta_{\text {div }}$ and $\sigma_{\mathrm{LT} . x}=L_{2} \sigma_{\mathrm{BW} . x}$, Eq. (62) can be further developed by inserting Eqs. (56), (59), and (60). Simple algebra leads us to

$$
\beta_{x} \approx\left[\left(\frac{L}{\phi L_{2}} \frac{\theta_{\mathrm{div}}}{\theta_{\mathrm{atm}, x}}\right)^{2}+1\right]^{-1 / 2} \text { if } W_{1} \ll L_{0},
$$

which implies that $\beta_{x}<1$. The case $W_{1} \gg c L_{0}$ leads to $\beta_{x} \ll 1$ with the expression

$$
\beta_{x} \approx \frac{c L_{0}}{W_{1}}\left[\left(\frac{L}{\phi L_{2}} \frac{\theta_{\mathrm{div}}}{\theta_{\mathrm{atm}, x}}\right)^{2}+2\right]^{-1 / 2} \quad \text { if } W_{1} \gg c L_{0} .
$$

Numerical values of $\beta_{x}$ and $\beta_{y}$ are displayed in Fig. 13 for a critical scenario, namely, the LEO-to-GEO link. $\theta_{\text {div }}$ is set to $3 \mu \mathrm{rad}$ and $\lambda=1 \mu \mathrm{m} . L_{0}=5 \times e^{h / 10 \mathrm{~km}}$. Care must be taken when evaluating $\beta_{x}$ or $\beta_{y}$. A beam of divergence $\theta_{\text {div }}$ of the order of $10 \mu \mathrm{rad}$ and emitted from a LEO satellite has a radius $W_{1}$ of the order of $L_{0}$. So using Eqs. (63) and (64) would lead in that case to inaccurate results. For Fig. $\underline{13}, \sigma_{\mathrm{BW}, x}$ and $\sigma_{\mathrm{BW}, y}$ were evaluated using a more general formula for $D_{w}$ based on Eq. (67). The plotted curves show the importance of the outer scale for beam-wander calculations. For each curve, one observes a maximum located in the stratosphere, not far from $20 \mathrm{~km}$ altitude. This maximum in the relative beam wander 


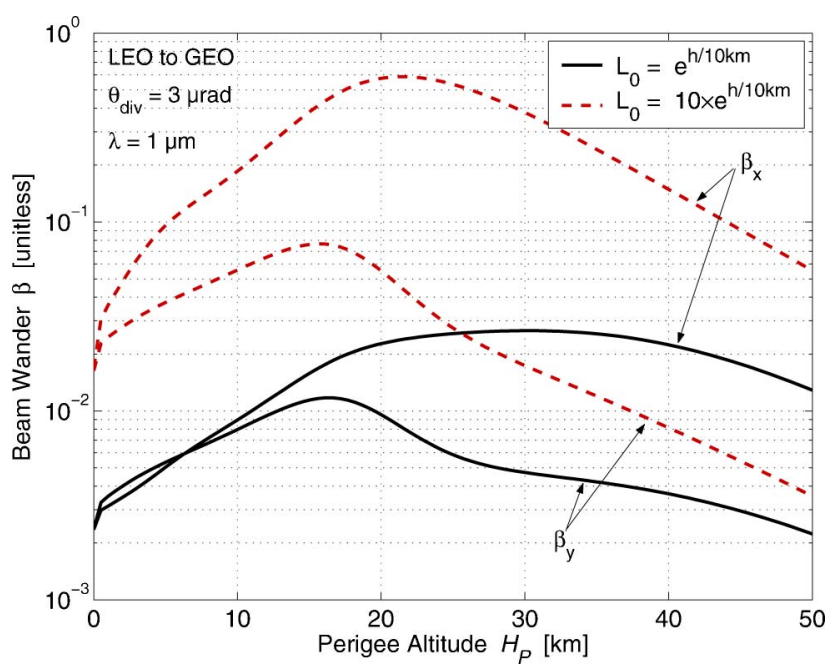

Fig. 13. (Color online) Relative beam wander $\beta_{x}$ and $\beta_{y}$ for a LEOto-GEO link with $\theta_{\text {div }}=3 \mu \mathrm{rad}$ and $\lambda=1 \mu \mathrm{m}$.

can be explained as follows. Starting at $H_{P}=0 \mathrm{~km}$ and going up, $\beta$ increases because $L_{0}$ increases and beam spread decreases (beam spread reduces the effect of absolute beam wander on relative beam wander). Above $H_{P} \sim 20 \mathrm{~km}$, even though $L_{0}$ still increases, $\beta$ starts decreasing because beam spread is negligible, whereas turbulence strength $\left(C_{n}^{2}, S_{n}^{2}\right)$ keeps decreasing. For $\beta<0.1$, beam wander may be regarded as negligible. We see that only the $x$ wander component combined with a large $L_{0}$ exceeds the value 0.1 .

\section{Effect on Optical Tracking}

Let us consider a terminal measuring AoA's over an aperture of diameter $D$ and transmitting its beam over the same aperture. The condition of Eq. (13) implies that the beam radius at the PS is much greater than the sending aperture, i.e., $W_{1} \gg D$. In terms of optical tracking, this situation corresponds to a strong on-axis aperture mismatch and leads to a decorrelation between the measured AoA and the angular deviation of the emitted beam [26]. Furthermore, the point-ahead angle, which is induced by the satellites' motions, strengthens this decorrelation [27]. So the reciprocity principle [28] does not apply in that context, and the tracking of the perturbed AoA will not improve the pointing performance. We can identify two situations where the atmosphere causes pointing errors:

i. The first situation is when the relative beam wander $\beta$ turns out to be significant (outer scale $L_{0}$ turns out to be large).

ii. The second situation is when $\beta$ is negligible but AoA fluctuations are tracked and are strong enough to displace the outgoing beam off the counter terminal. The AoA fluctuations are strong enough to cause a displacement if the condition $L \sigma_{\mathrm{AoA}} \ll$ $W_{\mathrm{LT}}$ is not fulfilled (for both $x$ and $y$ axes). The long-term beam radius is $W_{\mathrm{LT}}=s L \theta_{\text {div }}$, where $s$ is the beam spread factor, so that the condition becomes
$\sigma_{\mathrm{AoA}} \ll s \theta_{\text {div }}$. One can further develop the condition's expression using Eqs. (38), (56), and (57), and being careful about the link directions $\left(L_{1}\right.$ for the calculation of $\sigma_{\mathrm{AoA}}$ corresponds to $L_{2}$ for the calculation of $s$ ). For the critical LEO-to-GEO link, one can derive a simpler condition, namely, $\theta_{\text {atm }} \ll \theta_{\text {div }}$ for both $x$ and $y$ axes (considering $\phi=1$ for the $x$ axis is here a safety measure).

\section{Conclusion}

Based on given turbulence models, we have evaluated the spatial and temporal wave structure functions of optical ISLs occulted by the atmosphere. For such links, perturbation anisotropy is particularly pronounced. For certain scenarios, one can expect a coherence width $\rho_{0}$ smaller than the $\mathrm{Rx}$ aperture even above the cloud ceiling $\left(H_{P} \sim 13 \mathrm{~km}\right)$. ISL scintillation is generally the most severe disturbance above the cloud region. However, a distorted wavefront reaching the receiver can be an additional problem. Having evaluated the spatial WSF, we defined a diffraction angle $\theta_{\text {atm }}$ that is essentially determined by the path-integrated turbulence, with a weak dependence on the wavelength. The angular ratio $\theta_{\text {atm }} / \theta_{\text {div }}$ gives the strength of both the beam spread and the relative beam wander. We found that turbulence-induced beam spread and beam wander are significant for narrow beams $\left(\theta_{\text {div }}\right.$ of the order of $1 \mu \mathrm{rad}$ ).

\section{Appendix A}

The wave structure function of a Gaussian beam propagating through a thin phase screen was evaluated by Andrews and Phillips. Considering two points surrounding the beam center and a Kolmogorov spectrum, the WSF is given by Eq. (33), p. 656 of Ref. [17]. From this relation, we can derive the ratio of Gaussian-beam WSF $D_{\text {ga }}(\rho)$ to spherical-wave $D_{\text {sp }}(\rho)$, which yields

$$
\begin{aligned}
\frac{D_{\mathrm{ga}}(\rho)}{D_{\mathrm{sp}}(\rho)}= & \Gamma(11 / 6)\left[\frac{k\left(1-d_{3}\right)^{2} \rho^{2}}{4 \Lambda L d_{3}^{2}}\right]^{-5 / 6} \\
& \times\left\{{ }_{1} F_{1}\left[-\frac{5}{6} ; 1 ;-\frac{k\left(1-\bar{\Theta} d_{3}\right)^{2} \rho^{2}}{4 \Lambda L d_{3}^{2}}\right]\right. \\
& \left.-{ }_{1} F_{1}\left[-\frac{5}{6} ; 1 ; \frac{k \Lambda \rho^{2}}{4 L}\right]\right\},
\end{aligned}
$$

where ${ }_{1} F_{1}$ is the hypergeometric function and the dimensionless parameters $d_{3}, \bar{\Theta}$, and $\Lambda$ applied to our ISL scenario are given by

$$
\begin{aligned}
& d_{3}=L_{2} / L \\
& \bar{\Theta} \approx 1 \\
& \Lambda=2 L /\left(k W^{2}\right)
\end{aligned}
$$

We have $D_{\text {ga }}(\rho) \approx D_{\text {sp }}(\rho)$ under the two following conditions: in Eq. (65), the last argument of the first hypergeometric function tends to $-\infty$, and the last argument of the second hypergeometric function tends to 0 . These conditions can be written as 


$$
\begin{aligned}
& \frac{k L_{1}^{2} \rho^{2}}{4 \Lambda L L_{2}^{2}} \gg 1 \\
& \frac{k \Lambda \rho^{2}}{4 L} \ll 1
\end{aligned}
$$

and one easily finds that these two conditions are equivalent to Eq. (15).

\section{Appendix B}

This appendix is dedicated to the evaluation of the following integral:

$$
I_{p}=\int_{0}^{\infty} \frac{1-J_{0}(\kappa \rho)}{\left(\kappa^{2}+\kappa_{0}^{2}\right)^{p}} \kappa \mathrm{d} \kappa,
$$

which converges if $1<p<2$, or if $p \geq 2$ and $\kappa_{0} \neq 0$. This integral was evaluated by Lucke and Young [29] for the case $p=11 / 6$. Based on Eq. (11.4.44) of Ref. [30], $I_{p}$ takes the general form

$$
I_{p}=\frac{\kappa_{0}^{2(1-p)}}{2(p-1)}\left[1-\frac{2}{\Gamma(p-1)}\left(\frac{\kappa_{0} \rho}{2}\right)^{p-1} K_{p-1}\left(\kappa_{0} \rho\right)\right],
$$

where $K_{p-1}(\cdot)$ is the modified Bessel function of the second kind, order $p-1$. We deduce

$$
I_{p} \rightarrow \frac{\kappa_{0}^{2(1-p)}}{2(p-1)}, \quad \text { for } \kappa_{0} \rho \rightarrow \infty .
$$

To obtain the asymptotic behavior of $I_{p}$ for $\kappa_{0} \rho \ll 1$, we use the MacLaurin series given by Eq. (1.25) of Ref. [31]. This series leads to

$$
\begin{aligned}
\frac{2}{\Gamma(v)}\left(\frac{x}{2}\right)^{v} K_{v}(x)= & \sum_{n=0}^{\infty} \frac{(-1)^{n}}{n !}\left[\frac{\Gamma(-n-v)}{\Gamma(v)}\left(\frac{x}{2}\right)^{2 n+2 v}\right. \\
& \left.+\frac{\Gamma(-n+v)}{\Gamma(v)}\left(\frac{x}{2}\right)^{2 n}\right] .
\end{aligned}
$$

Based on Eqs. (67) and (69), one finds

$$
\begin{gathered}
I_{11 / 6} \approx \frac{3}{5} \frac{\Gamma(1 / 6)}{\Gamma(11 / 6)}\left(\frac{\rho}{2}\right)^{5 / 3}, \quad \kappa_{0} \rho \ll 1 \\
I_{11 / 6} \approx \frac{3}{5} \frac{1}{\kappa_{0}^{5 / 3}},
\end{gathered},
$$

The author thanks Y. Takayama, V. Sofieva, and C. Robert for helpful discussions.

\section{References}

1. W. B. Hubbard, J. R. Jokipii, and B. A. Wilking, "Stellar occultation by turbulent planetary atmospheres: a wave-optical theory including a finite scale height," Icarus 34, 374-395 (1978).

2. B. S. Haugstad, "Turbulence in planetary occultations, IV. Power spectra of phase and intensity fluctuations," Icarus 37, 322-335 (1979).

3. R. Woo, A. Ishimaru, and F.-Ch. Yang, "Radio scintillations during occultations by turbulent planetary atmospheres," Radio Sci. 15, 695-703 (1980).

4. R. Narayan and W. B. Hubbard, "Theory of anisotropic refractive scintillation: application to stellar occultations by Neptune," Astrophys. J. 325, 503-518 (1988).

5. R. Woo, "Spacecraft radio scintillation and solar system exploration," in Wave Propagation in Random Media (Scintillation), V. I. Tatarskii, A. Ishimaru, and V. U. Zavorotny, eds. (SPIE Press, 1993), pp. 50-83.

6. A. S. Gurvich and V. L. Brekhovskikh, "Study of the turbulence and inner waves in the stratosphere based on the observations of stellar scintillations from space: a model of scintillation spectra," Waves Random Media 11, 163-181 (2001).

7. A. S. Gurvich, V.Kan, and S. A. Savchenko, "Studying the turbulence and internal waves in the stratosphere from spacecraft observations of stellar scintillation: II. Probability distributions and scintillation spectra," Izv. Acad. Sci. USSR Atmos. Oceanic Phys. 37, 452-465 (2001).

8. A. S. Gurvich, V. F. Sofieva, and F. Dalaudier, "Global distribution of CT2 at altitudes $30-50 \mathrm{~km}$ from space-borne observations of stellar scintillation," Geophys. Res. Lett. 34, L24813 (2007).

9. Y. Takayama, T. Jono, Y. Koyama, N. Kura, K. Shiratama, B. Demelenne, Z. Sodnik, A. Bird, and K. Arai, "Observation of atmospheric influence on OICETS inter-orbit laser communication demonstrations," Proc. SPIE 6709, 67091B (2007).

10. A. S. Gurvich and M. S. Belen'kii, "Influence of stratospheric turbulence on infrared imaging," J. Opt. Soc. Am. A 12, 2517-2522 (1995).

11. M. S. Belen'kii, "Effect of the stratosphere on star image motion," Opt. Lett. 20, 1359-1361 (1995).

12. A. S. Gurvich and I. P. Chunchuzov, "Parameters of the fine density structure in the stratosphere obtained from spacecraft observations of stellar scintillations," J. Geophys. Res. 108 (D5), 4166 (2003).

13. J. W. Hardy, Adaptive Optics for Astronomical Telescopes (Oxford University Press, 1998).

14. G. C. Loos and C. B. Hogge, "Turbulence of the upper atmosphere and isoplanatism," Appl. Opt. 18, 2654-2661 (1979).

15. C. Robert, J.-M. Conan, V. Michau, J.-B. Renard, C. Robert, and F. Dalaudier, "Retrieving parameters of the anisotropic refractive index fluctuations spectrum in the stratosphere from balloon-borne observations of stellar scintillation," J. Opt. Soc. Am. A 25, 379-393 (2008).

16. F. Dalaudier, V. Kan, and A. S. Gurvich, "Chromatic refraction with global ozone monitoring by occultation of stars. I. Description and scintillation correction," Appl. Opt. 40, 866-877 (2001).

17. L. Andrews, R. Phillips, Laser Beam Propagation through Random Media, 2nd ed. (SPIE Press, 2005).

18. D. L. Fried, "Statistics of a geometric representation of wavefront distortion," J. Opt. Soc. Am. 55, 1427-1431 (1965).

19. Y. Cheon and A. Muschinski, "Closed-form approximations for the angle-of-arrival variance of plane and spherical waves propagating through homogeneous and isotropic turbulence," J. Opt. Soc. Am. A 24, 3478-3492 (2007).

20. Y. Takayama, National Institute of Information and Communications Technology (personal communication, 2008).

21. V. I. Tatarskii, The Effects of the Turbulent Atmosphere on Wave Propagation (Israel Program for Scientific Translations, 1971). 
22. R. F. Lutomorski and H. T. Yura, "Propagation of a finite optical beam in an inhomogeneous medium," Appl. Opt. 10, 1652-1658 (1971).

23. Y. Cai and S. He, "Average intensity and spreading of an elliptical Gaussian beam propagating in a turbulent atmosphere," Opt. Lett. 31, 568-570 (2006).

24. D. H. Tofsted, "Outer-scale effects on beam-wander and angleof-arrival variances," Appl. Opt. 31, 5865-5870 (1992).

25. L. C. Andrews, R. L. Phillips, R. J. Sasiela, and R. R. Parenti, "Strehl ratio and scintillation theory for uplink Gaussianbeam waves: beam wander effects," Opt. Eng. 45, 076001 (2006).

26. D. P. Greenwood, "Tracking turbulence-induced tilt errors with shared and adjacent apertures," J. Opt. Soc. Am. 67, 282-289 (1977).
27. S. Basu and D. Voelz, "Tracking in a ground-to-satellite optical link: effects due to lead-ahead and aperture mismatch, including temporal tracking response,"J. Opt. Soc. Am. A 25, 15941608 (2008).

28. D. L. Fried and H. T. Yura, "Telescope-performance reciprocity for propagation in a turbulent medium," J. Opt. Soc. Am. 62, 600-602 (1972).

29. R. L. Lucke and C. Y. Young, "Theoretical wave structure function when the effect of the outer scale is significant," Appl. Opt. 46, 559-569 (2007).

30. M. Abramowitz and I. A. Stegun, Handbook of Mathematical Functions (Dover, 1972).

31. R. J. Sasiela, Electromagnetic Wave Propagation in Turbulence; Evaluation and Application of Mellin Transforms, 2nd ed. (SPIE Press, 2007). 